\title{
MÉTODOS DE ANÁLISE DE PROJETOS HABITACIONAIS: UMA DISCUSSÃO SOBRE O MÉTODO I+D+VS
}

\author{
Methods of Analysis of Housing Projects: A Discussion \\ About the Method I+D+VS
}

\author{
Cássia Bartsch Nagle', Katrin Rappl', Leandro Silva Medrano²
}

RESUMO Os métodos de análise e avaliação de projetos habitacionais são importantes no auxílio do processo projetual e na melhora da qualidade das moradias. Nesse sentido, este artigo tem como objetivo apresentar e discutir um método de análise empregado no campo da habitação social: o método I+D+VS, do grupo de pesquisa NuTAC, Nuevas Técnicas, Arquitectura y Ciudad, da Universidade Politécnica de Madri. Apresenta-se também uma discussão sobre a necessidade e possibilidade de aplicação desse método no cenário brasileiro, apontando adaptações necessárias à realidade social, econômica, ambiental e cultural do país. Por fim, como pesquisa futura, incentiva-se a criação de um método de avaliação habitacional para a realidade brasileira, com a criação de softwares capazes de gerar gráficos e comparações entre os projetos, com a incorporação de valores aos parâmetros de análise do I+D+VS. Busca-se, portanto, ampliar a discussão sobre os métodos de análise e de avaliação de projetos habitacionais, auxiliar no aperfeiçoamento destes e contribuir para a sua aplicação na realidade brasileira.

PALAVRAS-CHAVE Método de análise, método de avaliação, habitação social.

ABSTRACT Methods of analysis and evaluation of housing projects are important in order to help the design process and improve the quality of dwellings. In this sense, this paper aims to present and discuss a method of analysis used in the field of social housing: the I+D+VS method, from NuTAC research group, Nuevas Técnicas, Arquitectura y Ciudad, from Polythechnic University of Madrid. The necessity and possibility of implementing it in the Brazilian scenario are highlighted in the paper, pointing necessary adjustments to social, economic, environmental and cultural aspects of the country. Finally, for future research, it encourages the creation of a housing evaluation method for the brazilian scenario, with a software capable of generating graphs and comparisons between projects with the incorporation of values to I+D+VS parameters. Therefore, we seek to increase the discussion on methods of analysis and evaluation of housing projects, also to improve them and contribute to their implementation in the Brazilian reality.

KEYWORDS Method of analysis, evaluation method, social housing.

Fonte de financiamento: FUNDAÇÃO DE AMPARO À PESQUISA DO ESTADO DE SÃO PAULO - FAPESP.

Conflito de interesse: Declaram não haver.

Submetido em: 02 dez., 2013 Aceito em: 24 dez., 2013 


\section{INTRODUÇÃO}

A habitação resulta de um processo cultural, histórico e técnico, com grande número de intervenientes e diversidade de funções, espaços, elementos, materiais e componentes. Apesar disso, as alterações exógenas ao ambiente doméstico nem sempre resultam em alterações drásticas em seus esquemas tipológicos tradicionais (MEDRANO, 2010).

Os diversos métodos de análise e de avaliação de projeto, criados ao longo dos anos para auxiliar o processo projetual, tiveram pouca inserção em países como o Brasil, onde os valores culturais e econômicos são, na maior parte das vezes, prioritários nas decisões de projeto. Apesar disso, salientamos a necessidade de aplicação dos deles no campo habitacional, com a finalidade de adotar melhores alternativas de projeto e contribuir para a reflexão sobre as questões habitacionais e os processos envolvidos, sobretudo nos casos das Habitações de Interesse Social (HIS), nas quais as decisões projetuais também operam na escala da política pública - de ampla inserção na sociedade e nas cidades.

Quando aplicadas ainda na fase de concepção do projeto, as análises e avaliações podem contribuir para a qualidade das habitações, atendendo da melhor forma as condições inerentes a projetos dessa natureza. Nesse sentido, o processo de análise, crítica e síntese possibilita a realização dos ajustes necessários ao projeto e à obtenção de melhores resultados.

Para identificar os principais métodos de avaliação disponíveis, procuramos revisar a literatura especializada, priorizando as revistas internacionais relevantes (QUALIS A1 e A2) com bom fator de impacto, e livros acadêmicos de grande repercussão na área de design methods. Destarte, consideramos os seguintes parâmetros: (a) livros publicados nos últimos 20 anos; (b) artigos de periódicos especializados publicados nos últimos 10 anos. Os periódicos consultados foram: Habitat International, Design Studies e Building and Environment (QUALIS A1 e A2 na avaliação da CAPES). Os artigos e livros resultantes dessa pesquisa são indicados na Tabela 1.

Tabela 1. Artigos QUALIS A1 e A2 e livros relacionados ao tema.

\begin{tabular}{|c|c|}
\hline Revistas & Artigos \\
\hline \multirow[t]{2}{*}{$\begin{array}{l}\text { Habitat } \\
\text { International }\end{array}$} & $\begin{array}{l}\text { Kowaltowski, Doris C. C. K.; Granja, Ariovaldo Denis. The concept of desired value as a } \\
\text { stimulus for change in social housing in Brazil. (2011) }\end{array}$ \\
\hline & $\begin{array}{l}\text { Mohit, Mohammad Abdul; Ibrahim, Mansor; Rashid, Yong. Assessment of residential } \\
\text { satisfaction in newly designed public low-cost housing in Kuala Lumpur, Malaysia. (2010) }\end{array}$ \\
\hline \multirow[t]{3}{*}{ Design Studies } & $\begin{array}{l}\text { Volker, Leentje; Lauche, Kristina; Heintz, John L.; Jonge, Hans de. Deciding about design } \\
\text { quality: design perception during a European tendering procedure. (2008) }\end{array}$ \\
\hline & $\begin{array}{l}\text { Dantec; Christopher A. Le; Dob, Ellen Yi-Luen. The mechanisms of value transfer in design } \\
\text { meetings. (2009) }\end{array}$ \\
\hline & $\begin{array}{l}\text { Cai, Hui; Yi-Luen Do, Ellen; Zimring, Craig M. Extended linkography and distance graph in } \\
\text { design evaluation: an empirical study of the dual effects of inspiration sources in creative } \\
\text { design. (2010) }\end{array}$ \\
\hline $\begin{array}{l}\text { Building and } \\
\text { Environment }\end{array}$ & $\begin{array}{l}\text { Kim, Sun-Sook; Yang, In-Ho; Yeo, Myoung-Souk; Kim, Kwang-Woo. Development of a } \\
\text { housing performance evaluation model for multi-family residential buildings in Korea. (2005) }\end{array}$ \\
\hline \multicolumn{2}{|r|}{ Livros } \\
\hline
\end{tabular}

ANTONCIC, R. P. A. Domicilio urbano. Santiago: ARQ, 2006.

LAWSON, B. How designers think: the design process demystified. 3rd ed. Oxford: Architectural, 1997.

MARTÍN BLAS, S.. I+D+VS: Futuro de la Vivienda Social en 7 Ciudades. Fundación Cultural COAM. Nov. 2011.

SAUNDERS, W. S. (ed.) Judging Architecture. Minneapolis: University of Minnesota, 2007.

Fonte: Elaborada pelos autores. 
A revisão indicada revela que, no âmbito da produção especializada contemporânea, são poucos os artigos relacionados ao tema. Ademais, muito deles apresentam metodologias de avaliação com indicadores relacionados às condicionantes "técnicas", como conforto, sustentabilidade, eficiência energética, economia, construtibilidade etc. As avaliações qualitativas, que buscam identificar as qualidades espaciais da habitação, tanto internamente quanto na relação com a cidade, são escassas.

Assim, para a elaboração de uma revisão consistente, ampliamos o recorte temporal da pesquisa a fim de identificar os principais autores relacionados ao tema. Procuramos nos restringir às três últimas décadas do século XX, período em que a atualização ou superação dos postulados canônicos do Movimento Moderno adquiriam maturidade teórica e conceitual.

Nesse sentido, uma das principais contribuições à área, no âmbito de nossa análise, tem início com os trabalhos de Alexander et al. (1977), com A Pattern Language, que colaborou para iniciar uma tentativa de análise de valores históricos e culturais na arquitetura, estabelecendo princípios de análise comparativa ao definir parâmetros de projeto, considerados ideais, aos quais novos projetos são comparados.

Os conceitos de Alexander (1977), os quais influenciam, principalmente, as culturas anglo-saxônicas, fundamentam grande parte das pesquisas que procuram aprimorar os métodos de projeto. Nesse sentido, Lawson (1997) indica que a análise projetual envolve a exploração de relações, procurando padrões na informação disponível, bem como a classificação dos objetivos. Outrossim, para esse autor, a avaliação de projetos deve levar em consideração tanto os critérios quantitativos quanto os qualitativos. A tentativa de reduzir o projeto a fatores quantitativos é insatisfatória, pois gera uma avaliação parcial, já que desloca o problema apenas para uma forma restrita de avaliação (LAWSON, 1997). Além disso, as variáveis que aparecem na tomada de decisão de projetos são tão diversas e complexas que a aplicação de um critério de valor aos parâmetros e conceitos envolvidos, com maior ou menor peso, não é suficiente para fundamentar uma decisão de projeto.

Atualmente é possível identificar diferentes metodologias que visam avaliar os projetos habitacionais. Tais metodologias fundamentam-se em critérios quantitativos, qualitativos e comparativos. Em geral, como descrito a seguir, os projetos são analisados por diferentes temas, tais como inserção urbana, composição, estética, sustentabilidade, tecnologia, sistemas construtivos, além de economia, diversidade, densidade, entre outros.

Por exemplo, Antoncic (2006) utiliza os seguintes parâmetros para análise e avaliação: relação com o contexto urbano; circulações e acessos; escala do edifício; tipos de convivência; parâmetros técnicos da unidade habitacional (materiais, conforto, instalações) e análise do agenciamento interno.

Além desta, destacam-se outras metodologias de análise e avaliação habitacional aplicadas na Europa, entre elas os métodos SEL e Qualitel.

O método SEL (Système d'évaluation de logements) foi utilizado na Suíça pela primeira vez em meados da década de 70 , com o intuito de promover a qualidade habitacional no país. De acordo com Sousa (1994), a avaliação é obtida pela relação entre custo e qualidade. Os 66 parâmetros utilizados para a análise quantitativa são divididos em três grupos: habitação, meio envolvente e local de implantação, os quais analisam a flexibilidade dos ambientes, a densidade, os equipamentos comunitários e a existência de serviços (sociais e culturais) no entorno.

Já o método Qualitel, introduzido na França em 1974, tem como objetivo possibilitar uma avaliação da habitação na fase de projeto e, assim, orientar o usuário na escolha da habitação em função de seus interesses. O método 
de avaliação é quantitativo e obtido por meio de uma pontuação em relação aos seguintes parâmetros: eficiência energética, acessibilidade, instalações, revestimentos e indicadores de conforto acústico (COSTA, 1995).

A coleta de dados nas análises quantitativas consiste em dois tipos de levantamentos, de arquivo e de campo (estes resumidos em cinco principais: observações, entrevistas, mapas mentais, questionários e levantamentos físicos - medições). Utilizam-se testes estatísticos, baseados na informação numérica tabulada numa planilha ou em gráficos, a partir de dados precisos. O objetivo da análise de dados é descrever, interpretar e explicar os dados coletados, de maneira que estes venham a responder às questões formuladas no estudo (REIS; LAY, 2005).

Entretanto, se interpretados e julgados, alguns métodos quantitativos, como questionários, podem ser utilizados para análises qualitativas do projeto, por meio de processos de percepção e cognição. A avaliação da qualidade de projeto relaciona-se diretamente à avaliação de desempenho de edificações e de espaços urbanos, através de seus usuários (REIS; LAY, 2006). A avaliação qualitativa de projetos habitacionais abrange inúmeros aspectos como estética, construtibilidade, flexibilidade, funcionalidade, manutenibilidade, adequação de acabamentos etc.

Neste artigo será tratado o método misto - quantitativo e qualitativo - do I+D+VS. O método desperta interesse por mostrar dados quantitativos mediados por análises qualitativas. Vale a pena ressaltar que ele não analisa o processo projetual, mas sim o projeto de habitação social em diferentes níveis: da escala urbana aos tipos de agrupações e sistemas construtivos.

\section{MÉTODO I+D+VS}

O método de análise I+D+VS é resultado do projeto de estudo Nuevas Técnicas y Vivienda Social do grupo NuTAC, da Universidade Politécnica de Madri, sobre habitação social contemporânea. O objetivo da pesquisa era estudar de que maneira as inovações e boas práticas poderiam ser introduzidas no campo da habitação. Somou-se, ainda, à necessidade de se analisar projetos em outras localidades. Segundo Martín Blas (2011), a habitação social está estritamente vinculada ao contexto social, econômico e político de cada cidade e país, porém isto não impede que cidades possam aprender com a experiência de outras.

A pesquisa analisou 8 edifícios em 7 cidades diferentes. São eles: Tetuán e Vara de Rey em Madri (Espanha), Can Travi em Barcelona (Espanha), Residência La Sècherie em Nantes (França), Hegianwandweg em Zurique (Suíça), Ciboga Terrain em Groninga (Holanda), Ljburg em Amsterdã (Holanda) e Kitagata em Gifu (Japão).

A investigação partiu da necessidade de se utilizar diferentes escalas de aproximação ao edifício, desde o entorno físico até os materiais e sistemas construtivos, para evitar observações parciais. Em cada projeto foram realizados quatro recortes que permitiram observar o edifício nesses contextos distintos:

1000x1000m: apresenta a relação entre a morfologia do entorno e o edifício (grau de continuidade ou fragmentação do tecido urbano).

250x250m: apresenta a organização interna básica, o tipo de agrupação das unidades e o seu contexto urbano imediato.

25x25m: indica a distribuição interna de uma unidade e sua relação com o restante do edifício.

1x1m: permite observar e descrever os materiais da construção e detalhes construtivos. 
Para analisar os projetos (Figura 1), foram utilizados: estrutura narrativa e códigos gráficos e numéricos. Além disso, o grupo de pesquisa (MARTÍN BLAS, 2011) adotadou os seguintes temas que estruturaram o método: diversidade, densidade e economia, os quais se relacionam com as escalas de amostragem.
Figura 1. Exemplo de um projeto analisado pela pesquisa. Fonte: Martín Blas (2011).

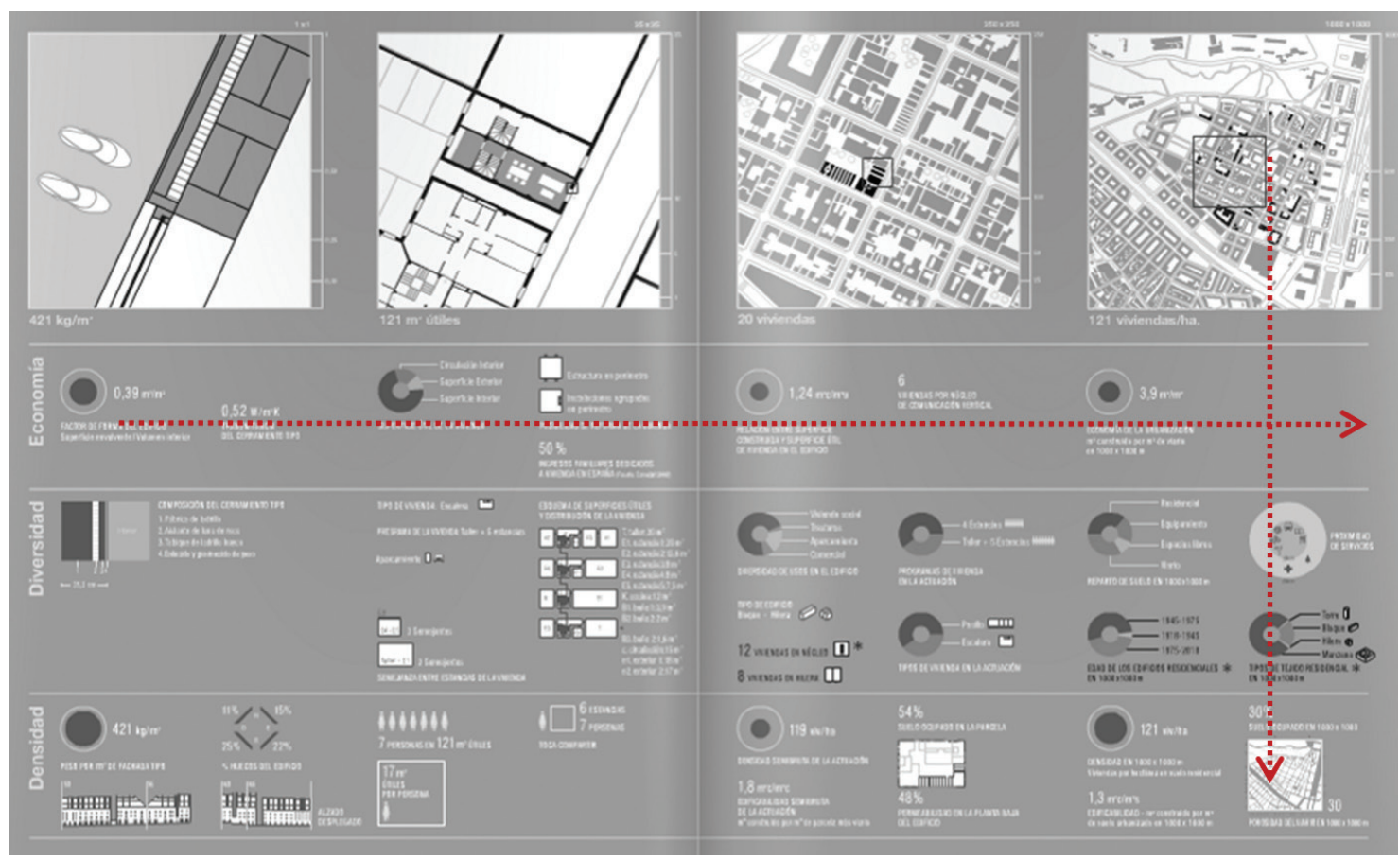

O item de economia expressa a relação de equilíbrio entre o uso de recursos e a satisfação de necessidades. $O$ tópico de diversidade evidencia a importância de uma combinação equilibrada entre atividades, grupos sociais, entre outros. Ressaltam-se ainda os estudos desenvolvidos por Jane Jacobs nos anos 60, sobre mescla de usos, permeabilidade urbana e definição de espaços públicos, que foram incorporados na análise. Já a densidade está fortemente relacionada ao tema de economia, e se refere à relação entre metros quadrados construídos, metro quadrado de solo e moradias por hectare. Também se relaciona com a diversidade urbana no sentido de estimular sua multifuncionalidade.

O método ressalta que deve-se olhar para estes pontos como fortemente vinculados e permeáveis. A seguir, serão apresentados os parâmetros analisados em cada tema (economia, diversidade e densidade) e em cada escala (1x1m, 25x25m, 250x250m e 1000x1000m).

\section{PARÂMETROS RELACIONADOS AO TEMA DE ECONOMIA (MARTÍN BLAS, 2011):}

- Fator de forma do edifício (1x1m): utilizado para avaliar o custo de condicionamento térmico no interior do edifício;

- Fator de transmitância do fechamento tipo (1x1m): relaciona inversamente este fator (superfície de fechamento externo) com a capacidade de isolamento dos materiais da mesma; 
- Relação entre superfície útil de distribuição (25x25m): relação entre área de circulação e distribuição interna (unidade);

- Possibilidade de reforma da moradia, com menores custos (25x25m): analisa a situação da estrutura e a concentração das instalações;

- Habitações que podem mudar de tamanho (25x25m): em porcentagem;

- Porcentagem da renda familiar destinada para a moradia (25x25m): permite contextualizar os dados econômicos do edifício analisado;

- Relação entre superfície construída e superfície útil de moradias no edifício (250x250m): avalia a economia do edifício. A superfície construída inclui as áreas internas das unidades e os espaços comuns;

- Número de habitações por núcleo de comunicações vertical (250x250m): dado repercute no custo total da construção, principalmente quando inclui a instalação de elevadores;

- Custo unitário do edifício (250x250m): custo médio por m²;

- Economia da urbanização (1000x1000m): coeficiente entre a área viária (ruas, praças, calçadas) e a área construída dos edifícios no entorno.

\section{PARÂMETROS RELACIONADOS AO TEMA DE DIVERSIDADE (MARTÍN BLAS, 2011):}

- Composição do fechamento tipo (1x1m): descrição dos materiais e espessuras;

- Tipo de habitações (25x25m): verifica a distribuição interna da unidade em tipo corredor, núcleo central, escada;

- Programa da habitação (25x25m): analisa a presença de usos que complementam a moradia, tais como, estacionamento, comércio;

- Semelhança entre áreas da habitação (25x25m): são consideradas como indícios de flexibilidade;

- Esquema de superfície útil e distribuição (25×25m): esquema de habitação tipo e acessos;

- Diversidade de usos no edifício (250x250m): comparação entre área de usos distintos (habitação, comércio, serviços) dentro do edifício;

- Tipo de edifício (250x250m): tipo de implantação do edifício, tais como torre, quadra, linha, isolado;

- Tipo de agrupações (250x250m): agrupamento das unidades habitacionais, tais como corredor, núcleo, linha;

- Programa das unidades no edifício (250x250m): verifica a possibilidade de atender diversos perfis de moradores;

- Tipos de habitações no edifício (250x250m): analisa a distribuição interna das habitações no edifício;

- Análise de entorno (1000x1000m): analisa a mescla de usos, atividades, tipos de edifícios e usuários;

- Idade dos edifícios residenciais (1000x1000m): analisa se é uma área urbana consolidada ou em crescimento;

- Proximidade de serviços (1000x1000m): verifica a proximidade com equipamentos de serviço e transporte público;

- Tipos de tecido residencial (1000x1000m): identifica os tipos de implantações urbanas no entorno.

\section{PARÂMETROS RELACIONADOS AO TEMA DE DENSIDADE (MARTÍN BLAS, 2011):}

- Peso por metro quadrado de fachada tipo (1x1m): soma das densidades de cada uma das camadas (materiais) por sua espessura;

- Indicação de cheios e vazios nas fachadas (1x1m): análise gráfica em relação a cada orientação; 
- Porcentagem de aberturas em cada orientação (1x1m): relação entre área da fachada e área das aberturas;

- Relação entre habitantes e áreas da unidade (25×25m): relação entre a superfície útil interna e o número de pessoas (por unidade);

- Densidade semibruta (250x250m): relação entre o número de habitações do edifício e a área do lote mais a parte proporcional da via;

- Índice de edificabilidade (250x250m): relação entre área construída e área do lote mais a parte proporcional da via;

- Solo ocupado no lote (250x250m): porcentagem

Figura 2. Comparação dos recortes. Fonte: Martín Blas (2011). de solo ocupado;

- Permeabilidade na planta baixa do edifício (250x250m): indica o grau de relação do edifício com o espaço público ao seu redor;

- Densidade em solo residencial (1000x1000m): número aproximado de habitações por hectare no entorno urbano;

- Índice de edificabilidade (1000x1000m): relação entre área edificada e o tecido urbano da amostra, incluindo todos os usos da área urbana;

- Solo ocupado (1000x1000m): considera que a ocupação indica o grau de compacidade do tecido urbano;

- Porosidade viária (1000x1000m): número de vias que cortam o perímetro da amostra. $\mathrm{O}$ dado fornece informações sobre a densidade do tecido urbano e também sobre o tamanho das quadras.

Por fim, o método apresenta os resultados em gráficos que comparam os 8 projetos. Seguem alguns exemplos:

A comparação entre os recortes $1000 \times 1000$ m (Figura 2) fornece informações sobre o grau de compactação do tecido urbano entre os diferentes projetos. A amostra mais compacta é do projeto Vara del Rey no centro histórico de Madri, e a mais dispersa é do projeto Can Travi, em

Figura 3. Diversidade de usos. Fonte: Martín Blas (2011). Barcelona.

A análise de diversidade de usos (Figura 3) relaciona as porcentagens de usos não residenciais no edifício com seu entorno. Trata-se de avaliar como o edifício responde ao seu entorno, aumentando ou diminuindo a porcentagem de usos não residenciais na área. São apresentados, ainda, gráficos que apresentam a relação entre índice de edificabilidade e ocupação, a qual permite conhecer a solução volumétrica adotada pelo edifício, o do grau de relação da edificação com o espaço público, entre outros.

\section{LACUNAS NO MÉTODO I+D+VS}

Pode-se verificar que este método exige um estudo aprofundado dos projetos para a coleta de dados. Entretanto, muitas vezes estes dados não são divulgados ou não são facilmente acessados pelos pesquisadores.

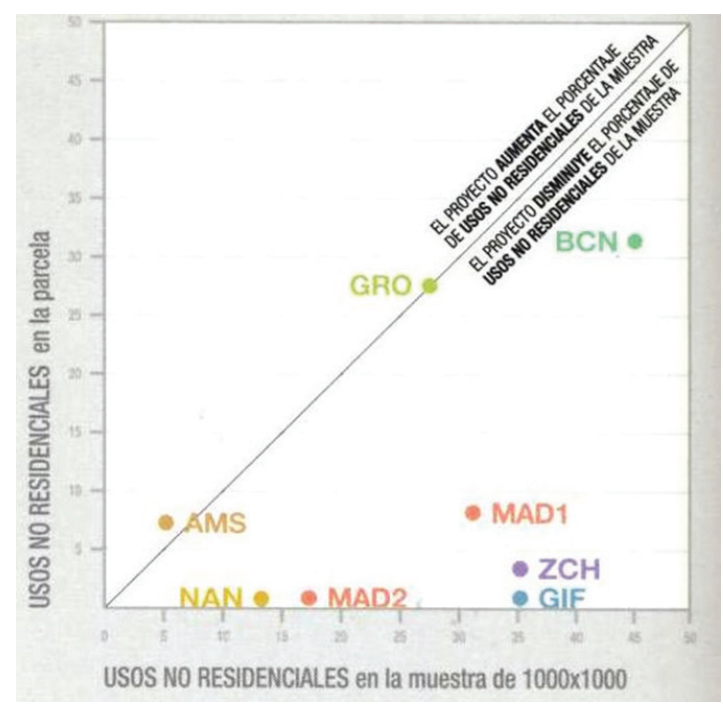


Ademais, percebemos que este não é um estudo completo do projeto, uma vez que algumas questões não são consideradas, ou, em alguns casos, são tratadas como parte de outros tópicos, aparecendo no estudo de forma superficial, tais como acessibilidade, sustentabilidade, legislação, conforto ambiental, estética, infraestrutura, segurança e durabilidade.

A seguir, destacamos alguns itens não considerados pelo método I+D+VS (Tabela 2):

Tabela 2. itens não contemplados pelo método I+D+VS.

\begin{tabular}{|c|c|c|}
\hline Item & Escala & Itens não considerados \\
\hline \multirow[t]{3}{*}{ Sustentabilidade } & $25 \times 25 m$ & $\begin{array}{l}\text { - análise do uso de energia limpa; } \\
\text { - consumo de energia comparada à produção de energia renovável } \\
\text { (energia solar); } \\
\text { - orientação do edifício; } \\
\text { - iluminação e ventilação naturais; } \\
\text { - materiais e tecnologias sustentáveis utilizadas; }\end{array}$ \\
\hline & $250 \times 250 \mathrm{~m}$ & $\begin{array}{l}\text { - sombras causadas de acordo com a orientação solar; } \\
\text { - permeabilidade urbana; } \\
\text { - arborização; }\end{array}$ \\
\hline & $1000 \times 1000 \mathrm{~m}$ & $\begin{array}{l}\text { - existências e disposição dos modais de transporte; } \\
\text { - proximidade de serviços e comércio; } \\
\text { - existência de ciclovias ou a possibilidade de aluguel de bicicletas; } \\
\text { - coleta e manejo do lixo das residências; }\end{array}$ \\
\hline \multirow[t]{3}{*}{ Infraestrutura } & $25 \times 25 m$ & $\begin{array}{l}\text { - quantidade e disposição das vagas de estacionamento; } \\
\text { - provisão de água potável; } \\
\text { - coleta de esgoto dentro dos edifícios; }\end{array}$ \\
\hline & $250 \times 250 \mathrm{~m}$ & $\begin{array}{l}\text { - área permeável no lote; } \\
\text { - pontos de parada das linhas de transporte público; } \\
\text { - equipamentos públicos existentes; } \\
\text { - drenagem e coleta de água; } \\
\text { - existência de praças e áreas verdes; }\end{array}$ \\
\hline & $1000 \times 1000 \mathrm{~m}$ & $\begin{array}{l}\text { - análises de transporte público e dos equipamentos públicos } \\
\text { (creches); } \\
\text { - existência de estações de tratamento de água e esgoto; } \\
\text { - existência de parques; }\end{array}$ \\
\hline \multirow[t]{3}{*}{ Acessibilidade } & $25 \times 25 m$ & $\begin{array}{l}\text { - adequação dos desníveis ao programa; } \\
\text { - questões de funcionalidade e ergonomia; }\end{array}$ \\
\hline & $250 \times 250 \mathrm{~m}$ & $\begin{array}{l}\text { - relação entre as unidades acessíveis e as não acessíveis; } \\
\text { - largura de calçadas; } \\
\text { - existência de obstáculos nos caminhos; } \\
\text { - indicações e visibilidade de percurso; } \\
\text { - vagas para deficientes próximas à entrada; }\end{array}$ \\
\hline & $1000 \times 1000 \mathrm{~m}$ & $\begin{array}{l}\text { - análise dos problemas de percurso (obstáculos existentes, } \\
\text { desníveis); } \\
\text { - acesso ao transporte público. }\end{array}$ \\
\hline
\end{tabular}

\section{APLICAÇÃO DO MÉTODO EM PROJETOS NO BRASIL}

Atualmente no Brasil identifica-se o aquecimento do mercado imobiliário incetivado principalmente pelo Programa "Minha Casa, Minha Vida", do governo federal. A construção de habitações com o objetivo de diminuir 
o déficit habitacional no país é uma das prioridades deste programa, que ampliou sua meta inicial para 2 milhões de casas até 2014, com o investimento de 71,7 bilhões de reais (BRASIL, 2008).

Em relação ao volume de recurso público, o programa possibilitou um grande investimento orçamentário na área habitacional (BONDUKI, 2008). Diante da facilidade na obtenção de subsídios para a construção, verifica-se a multiplicação de grande quantidade de residências e empreendimentos, principalmente em áreas periféricas das cidades, locais nos quais o valor do solo é mais barato e a falta de infraestrutura e serviços é recorrente.

Além disso, a falta de qualidade urbana existente na relação entre habitação social e cidade nesses novos emprendimentos é uma realidade a ser destadaca. Conforme afirma Ferreira (2012), novos bairros surgem no país aparentemente sem preocupação com a qualidade urbana, alinhando casas idênticas ou enfileirando torres habitacionais com baixo padrão construtivo. Outros autores, tais como Rolnik et al. (2010) e Maricato (2009), complementam esta ideia ao destacarem a importância da localização desses empreendimentos em áreas com infraestrutura adequada e próximos a locais com oferta de trabalho.

Nesse contexto, consideramos de extrema importância a relação do edifício com seu entorno, além da provisão de infraestrutura e serviços disponíveis para os moradores. Por isso, diante da realidade que enfrentamos no país, torna-se necessária a busca por métodos que auxiliem na avaliação da produção habitacional e na sua relação com a cidade, a fim de que metodologias e soluções projetuais sejam aprimoradas.

Sendo assim, a adaptação do método à realidade social, econômica, ambiental e cultural brasileira é importante. Para que ele possa ser aplicado no país, algumas alterações e alguns complementos precisariam ser feitos. Por exemplo, no Brasil, as condições ambientais, como insolação e temperaturas médias são diferentes dos países em que o método apresentado foi aplicado. Além disso, no contexto nacional, muitos dos dados necessários para a análise com o método I+D+VS não são divulgados ou não estão disponíveis para consulta, o que dificulta ou mesmo impossibilita a análise de alguns itens.

Um dos pontos mais importantes a ser levado em consideração é o tipo de habitação social produzida no Brasil. As configurações espaciais, a situação urbana, a realidade econômica e as técnicas construtivas se diferem muito dos modelos encontrados nos países europeus, para os quais o método em análise foi desenvolvido. Enquanto que na Europa as HIS têm média ou alta densidade, estão inseridas no tecido urbano das cidades, procuram valorizar a qualidade construtiva e adotam valores próximos às habitações de mercado, no Brasil, as HIS ainda são precárias, de baixa densidade e alijadas das áreas de urbanização consolidada.

Portanto, o método espanhol, se aplicado à realidade nacional, precisaria ser adaptado para incorporar características não contempladas no estudo original, como, por exemplo, a medição da distância das áreas residenciais ao centro das cidades em que se encontram, assim como dados relacionados à oferta de serviços públicos oferecidos pelo governo dentro de cada conjunto residencial, que podería ser incorporado ao parâmetro já existente "proximidade de serviços" (escala 1000x100m).

O tipo de financiamento também é um item importante a ser considerado (por exemplo, se é de promoção pública, particular, aluguel social ou aluguel comum). $\mathrm{O}$ entendimento da relação entre o projeto e as políticas públicas vigentes auxilia na compreensão de diversos itens adotados no projeto.

Destacamos, ainda, em relação à realidade brasileira, a necessidade da criação de uma metodologia de avaliação que possa ser aplicada, como 
método complementar, nos concursos de arquitetura. Nos últimos anos, foram muitos os concursos de arquitetura dedicados às HIS, como o Habitasampa, o Morar Carioca, o Prêmio Caixa-IAB e o Renova São Paulo. Pesquisas recentes demonstram que nem sempre seus resultados foram condizentes com os objetivos dos promotores. Ademais, não representaram, necessariamente, soluções inovadoras, capazes de contribuir para a evolução desse campo disciplinar (MEDRANO; SPINELLI, 2014).

\section{CONCLUSÃO}

Pode-se verificar, portanto, a partir do estudo do método do I+D+VS, que alguns critérios de análise poderiam ser adicionados, a fim de torná-lo mais eficiente, principalmente no que se refere às questões urbanas e sociais vinculadas às HIS. Também os temas relacionados aos aspectos históricos e culturais são fragilizados neste método. Por exemplo, não se avalia a participação da comunidade nos projetos, nem as relações de identidade. As análises de inovações ponderadas a partir do ponto de vista histórico e do contexto local também não recebem a devida importância.

Além disso, no caso de aplicação do I+D+VS a projetos brasileiros, algumas considerações relacionadas às condicionantes ambientais, urbanas, à infraestrutura existente, ao tempo de deslocamento da periferia ao centro, ao tipo de financiamento e às políticas vigentes deveriam ser consideradas.

Como proposta de pesquisa futura, sugere-se a criação de um método de avaliação adequado à realidade brasileira, que auxilie no desenvolvimento de projetos de HIS no país, assim como na relação entre a arquitetura habitacional e a cidade. Este método de avaliação, por sua vez, poderia ser automatizado através de softwares capazes de gerar os gráficos propostos. Os softwares poderiam, ainda, para efeito de comparação dos resultados obtidos, sobrepor os gráficos e desenhos resultantes da análise dos diversos projetos, indicando quais destes projetos estariam dentro de uma faixa de resultados considerada ótima ou aceitável.

Por fim, espera-se com esta pesquisa ampliar a discussão sobre métodos de análise e avaliação de projetos, assim como auxiliar no estabelecimento de diretrizes metodológicas que possibilitem a melhora na qualidade dos projetos habitacionais.

\section{AGRADECIMENTOS}

Fundação de Amparo à Pesquisa do Estado de São Paulo (FAPESP). Processos n 2012/12146-7 e 2012/12321-3.

\section{REFERÊNCIAS}

ALEXANDER, C. et al. A pattern

language: towns, buildings, construction. New York: Oxford University, 1977.

ANTONCIC, R. P. A. Domicilio urbano. Santiago: ARQ, 2006.

BONDUKI, N. Política habitacional e inclusão social no Brasil: revisão histórica e novas perspectivas no governo Lula. Revista Eletrônica de Arquitetura e urbanismo, São Paulo, n. 1, p. 70104, 2008.

BRASIL. Ministério das Cidades. Lei Federal 11.977/2008. Programa Minha
Casa Minha Vida. Diário Oficial da República Federativa do Brasil, Brasília, DF, 2008.

COSTA, J. M. Métodos de avaliação da qualidade de projectos de edifícios de habitação. 1995. Dissertação (Mestrado)Universidade do Porto, Porto, 1995.

FERREIRA, J. S. W. Produzir casas ou construir cidades? Desafios para um novo Brasil urbano: parâmetros de qualidade para a implementação de projetos habitacionais e urbanos. São Paulo: FUPAM, 2012. 
LAWSON, B. How designers think: the design process demystified. 3rd ed. Oxford: Architectural, 1997.

MARICATO, E. O nó da terra urbana. Carta Capital, São Paulo, n. 570, p. 45, 2 set. 2009.

MARTÍN BLAS, S. I+D+VS: Futuro de la Vivienda Social en 7 Ciudades. Fundación Cultural COAM, Nov. 2011.

MEDRANO, L. Habitação coletiva e cidade. Invenção sem ruptura. Pós. Revista do Programa de Pós-Graduação em Arquitetura e Urbanismo da FAUUSP, São Paulo, v. 17, n. 27, p. 5471, 2010. http://dx.doi.org/10.1590/S151895542010000200005

MEDRANO, L.; SPINELLI, J. Urban policies and projects for social housing in central areas. The case of the Habitasampa competition (São Paulo, Brazil). Habitat International, Oxford, v. 41, p. 3947, 2014. http://dx.doi.org/10.1016/j. habitatint.2013.10.004
ROLNIK, R. et al. (Coords.). Como produzir moradia bem localizada com recursos do programa minha casa minha vida? Implementando os instrumentos do Estatuto da Cidade. Brasília: Ministério das Cidades, 2010.

REIS, A. T. L.; LAY, M. C. D. Análise quantitativa na área de estudos ambientecomportamento. Ambiente Construído, Porto Alegre, v. 5, n. 2, p. 21-36, abr./ jun. 2005.

REIS, A. T. L.; LAY, M. C. D. Avaliação da qualidade de projetos - uma abordagem perceptiva e cognitiva. Ambiente

Construído, Porto Alegre, v. 6, n. 3, p. 21 34, jul./set. 2006.

SOUSA, M. J. C. N. C. Aplicação do método SEL a projectos de edifícios da habitação elaborados em Portugal. 1994. Dissertação (Mestrado)Universidade do Porto, Porto, 1994.

\section{Correspondência}

Cássia Bartsch Nagle, cassiabn@uol.com.br

Katrin Rappl, katrappl@gmail.com

Leandro Silva Medrano, medrano@fec.unicamp.br 